\title{
Estudio electroencefalográfico en diabéticos juveniles
}

\author{
Dres. Irig Melia G.. Maria E. Godoy B., Manuel Garcia de los Rím A. y Juan C. Tapia (;.
}

\begin{abstract}
We carried out electroencephalogram (EEG) over anamestic hypodycemias in 100 jusenile diabetics and in 50 conirols.

The following outcomes were revealed by the electroencepbalographic tracings: $69 \%$ normal; $12 \%$ within standard accep1able limits iCerebral Bioelectrical Inmaturity 8\%; Low Convulsive Threshuld 2\%; Slow Temporal Focus 2\%; und 19\% absolutely altered (Interictal Period of Generalized Epilepsy 12\% and Inespecificsl Difuse Neuronal Damage 7\%?. There was no pathological EEG in the control group.

Interictal periud of generalized epilepsy was noticed more frequently ( $39 \%$ ) among patients under 10 years old, and wiht a short evolution of diabetes $(4+2,4$ years! and not related with anamnestic hypuglycemias.

Only children over 11 years old with a long evolution of diabeles $(13,3+7,9$ gears $)$ sppeared to have inespecifical difuse neuronal damage. All of them had frequent slight hypogiycemias in their background and the great majority had suffered hypoglycemic shock. This kind of EEG was not associated with the presnce of hypoglycemic convulsions.

We found no relation between obnormal outcomes of EEGs and the previous glyceruic levely ur with the usual metabolic control of the patient.

A9 a conclusion 19\% of the studied jurenile diabetics presents an allefed EEG. Epilepsy is not related with the duration of diabetes, neither with the lype of bypoglycemia. The inespecifical difuse neurunal damage arises when the diabetes is older and the patient more mature and seems to be, at least partially, favored by different forms of hypoglycemia.
\end{abstract}

Es un hecho conocido que las hipoglicemias repetidas y profundas o prolongadas por falta de tratamiento oportuno y eficaz, pueden originar daño cerebral definitivo y en algunos casos provocar la muerte. (1-2)

Algunos autores han demostrado en pacientes insulinodependientes con hipoglicemias a repetición, una mayor frecuencia de electroencefalogramas (EEG) anormales. Así, Greenblatt 3) encontró en diabéticos con estas características que el $51 \%$ tenían EEG alterados. Log trabajos de Gardner y Reyerbach '4' también apoyan estos hallazgos y sostienen además que las hipoglicemias provocarían trastornos de la personalidad por lesión cerebral.

Jakobson's' estudió 40 diabéticos insulinodependientes, 22 estables y 18 inestables, y encontró que estos últimos tenían mayores alteraciones del EEG, en espetial de tipo paroxistico; considera que

Departamenku de Nutririón y Diabetes. Serrifio y Linidad Dir

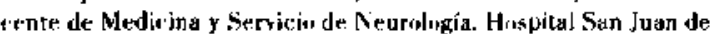
Dius. Faculıad de Moalieina. Sede Occidente. Universidad de thile. factores cerebrales pueden contribuir a la labilidad de la diabetes.

Eeg-Olofsson ${ }^{16:}$ en un completo trabajo publicado recientemente, plantea la posibilidad de que los diabéticos juveniles tengan una encefalopatía diabética primaria. con una sensibilidad individual a reaccionar con síntomas neurológicos frente a una hipoglicemia, debido a un trastorno cerebral congénito o adquirido.

El interés de este tema aún controvertido y no bien definido nos motiví a investigar las características electroencefalográficas en un número importante de diabéticos juveniles, a fin de conocer la pusible repercusión de diversas formas y grados de hipoglicemias anamnésticas en este tipo de registro cerebral.

\section{MATERIAL Y METODO}

Se esludió el EEG en 100 diabéticos juveniles, insulinodependientes, elegidos al azar entre los atendidos labitualmente en el Servicio y en 50 stejetos 
contrules, aparentemente samos, de sexu y edades promedis stomejantes a los diabélicas.

Todos los pacientes fiueron sometidos a una encuesta standarizada sobre antecedentes hipoglicemicos, clasificandsse en la siguiente forna: 1) Sin hipoglicemia. 2) Síntumas leves o maderados ocasiomales !l vez al mes). 31 Leves " moderadus frecuentes. 4) Convulsiones. 5) Shuck hipuglicénico. En la mañatla, momentos antes de hacer el EFG, se les practićs glicemia, a fin de realizar esle examen con niveles de glucosa sanguinca superiores a $100 \mathrm{mg} \%$.

El estudi" electroencefalográfico se efectuci en al Servicio de Neurologia, utilizándose un electroencelalógrafo Alvar de 8 canales con corriente alterna de 220 velties, con una amplificacien de 50 u y una velotidad de $30 \mathrm{~mm}$ por segundo. Se registró la rorriente neuronal por medio de electrodos adherentes subre la cabeza del paciente, según la técnica staudard de ubtener las derivaciones (Métodu $10 \times$ 20\% del acuerdo de la Federación Internacional para distancia de colocación de electrodos) y se registraron derivaciones bipolares. El EEF se realjzó en condicisnes standard de reposo, vigilia. blaques cortico visual y activado mediante la maniobra de hiperventilación.

Los EEG se clasificaron en la siguiente forma:

1) Noniwales (N).

2) Tensionales (1). Con taquirritmia de actividad muscular.

3) Inmadurez Bioeléctrica Cerebral (IBC). Actividad neuronal que conserva el ritmo de base. pero parcialmente reemplazada per desorganización theta $y$ a veces delta, de distribución difusa en ambus lemisferios, ocasionalmente bioccipital que se activa con la maniobra de hipervenlilación y vuelve a las condiciones basales al minuto de finalizada esta maniobra.

4) Cmbral Convtulsitante Bajo (LCB). Es el registro electroencefalográfico standard normal que, activado con la maniobra de hiperventilación, vuelve a las condiciones basales después del minuto de finalizada la maniobra, en pacientes mayores de 25 años de edad.

5) Foco Temporal Lento (1' $\left.\mathrm{f}^{2} \mathrm{~L}\right)$. La actividad lenta theta delta se mantiene durante lodo el regisIro en regiones temporales, conservándose el resto del trazado con actividad normal.

6) Intercrítico de comicialidad jeneralizada [ICG). El registro electroencefalográfico está reemplazado parcialmente pur actividad lenta theta delıa. interrumpido to varias ocasiones por paroxismos de estos mismos elementos lentos, bilaterales, asincri- nicos. asimétricens, de altu voltajo y de durarion varialile..

7) Sufrimiento Neurothal Difuso Inespecifico (SNDI). La actividad basal se erseuentra parcial o Intalmente remplazada por lentitud theta delta, de distribucion difusa en ambus bemisferios y que se mantiene durante todo el registro, no modificándose con la maninbra de hiperventilarion.

El material se distribuye en 39 hombres y 61 mujeres. Todus los casos iniciaron su enfermedad antes de los 15 años de edad y el $63 \%$ de ellos antes de los 10 añus. ('Tabla N."1.)

\section{Tabla N. ${ }^{0} 1$}

Distribución de pacientes por sexo y edad de diagnostico.

\begin{tabular}{rrrr}
\hline Edad (años) & Hombres & Mujeres & Total \\
\hline 5 & 8 & 9 & 17 \\
$6-10$ & 15 & 31 & 46 \\
$11-15$ & 16 & 21 & 37 \\
\hline Total & 39 & 61 & 100
\end{tabular}

La distribución de los pacientes segun edad aparece en la Tabla N."2. Se observa que en la mayoría de las décadas existe un porcentaje semejante de hombres y mujeres y que el $58 \%$ de los casos tienen entre. 11 y 20 años de edad, con un rango entre 3,5 y 36 años.

\section{Tabla $\mathbf{N}^{\circ} \mathbf{2}$}

Distribución de pacientes por sexo y edad actual.

\begin{tabular}{rrrrrrr}
\hline \multirow{2}{*}{ Edad actual } & \multicolumn{2}{c}{ Hombres } & \multicolumn{2}{c}{ Mujeres } & \multirow{2}{*}{ Total } \\
& N. & \% & \multicolumn{2}{c}{ N. $^{\circ}$} & $\%$ & N. \\
\hline 10 & 3 & 8 & 10 & 16 & 13 \\
$11-20$ & 26 & 67 & 32 & 52 & 58 \\
$21-30$ & 9 & 23 & 15 & 25 & 24 \\
31 & 1 & 2 & 4 & 7 & 5 \\
\hline Total & 39 & 100 & 61 & 100 & 100
\end{tabular}

El 62\% de los enfermos tenían una diabetes de menos de 10 años de evolución y el $38 \%$ más de 10 años. El grado de control metabúlico promedio de los parientes fue: $7 \%$ bueno, $28 \%$ regular y $65 \%$ malo, de acuerdo a la clasificación utilizada en el Departamento.1? 
Existia el antecedentr de roma tuetoracidutico . 1 1. $34 \%$ del material. En relation a la existencia del sindrome diabétiancrónicos posdemos señalar que el $22 \%$ tenia un grado variable de Retinepatia (9\% proliferantei y $11 \%$ de Nefroputia, ningún casol presentaba insuficiencia renal.

\section{RESLLTADOS}

La distribución de los resultados de los EEC aparere en la Figura N." 1. Se puede observar que el $52 \%$ sor Normales y se reparten de manera casi homengenea en ambos sexos. Los Tensionales se presen-

\section{FIGURA NO 1.- RESULTADD OEL ELECTROENCEFALOGRAMA}

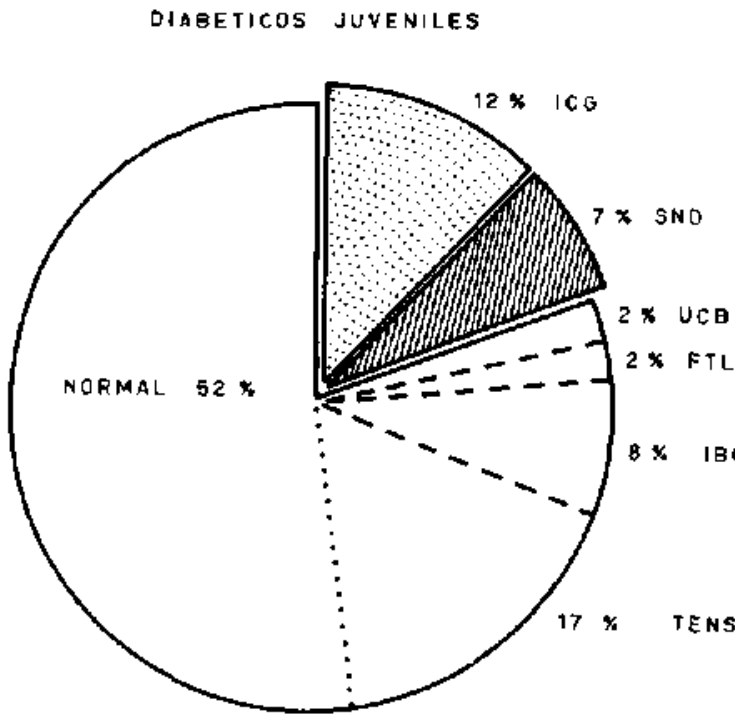

taron en el $17 \%$ y corresponden principalmente a las mujeres; la Inmadurez Bioeléctrica Cerebral (8\%) se encontró con mayor frecuencia en los hornbres: el Umbral Convulsivante Bajo se observó sólı en un hombre y una mujer. En dos casos hubo Focu Temporal Lento (un hombre y una mujer): los Intercríticos de Comicialidad Generalizada (12\%) están presentes tres veces más en las mujeres y el Sufrimientu Neuronal Difuso Inespecifico (7\%) se distribuye por igual en ambos sexos. En resumen, encontramıs un $69 \%$ de EEG Normales ( $N$ y $\mathrm{T}$ ), 12\% dentro de limites normales (FTL. IBC y CCB) y 19\% francamente anormales (ICG y SNDI). En el grupocontrol, el $78 \%$ de los EEG fue Normal, el $8 \%$ Tensional y el 14\% presentó Inmadurez Bioeléctrica Cerebral, no encontrándose ningún EEG patológico.

En la Tabla N.o 3 se presentan los resultados de los EEG según la edad actual de los pacientes, observándose que los trazados cun carácter de Inmadurez se encontraron exclusivamente en los menores de 20 años. La Comicialidad aparece con mayor
CONTROLES

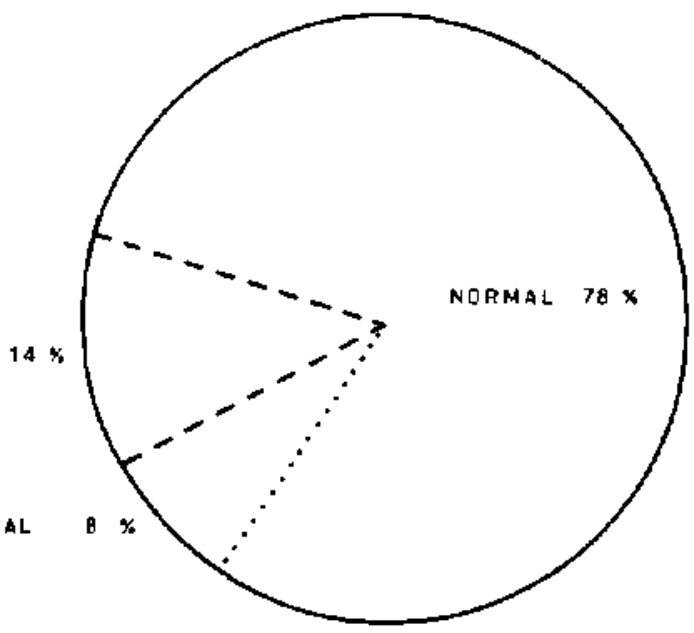

frecuencia (39\%) en los menores de 10 años y suín en el sexo femenino, disminuye al $10 \%$ en el grupo de 11 - 20 añus y se encontró sólo un caso después de los 21 años de edad. Existe significancia estadística ( $P<0.05$ ) sólu en el grupu de menes edad, con respecto a los otros dos.

El porcentaje más alto de curvas electruencefalográficas con SNDl se observó en los mayores de 21 años ( $14 \%)$, que baja a $5 \%$ entre los $11-20$ añus. no existiendu ningún caso en los menores de 10 años; sin embargo, no hay diferencias estadisticamente significativas entre estos porcentajes.

Al relacionar los distintos tipos de EEG y la antigüedad de la diabetes, se destaca que los trazados Normales y Tensionales tienen una distribución uniforme en los pacientes con menos de 10 años y con más de 10 años de evolución de la diabetes. La Inmadurez Bioeléctrica Cerebral se observó en una frecuencia tres veces mayor en el grupo con menos añus de enfermedad. La Comicialidad se encontró sólo en el grupo de menor evolución $(4$ años $\pm 2,4)$. 
Las curvas con S.VDI aparecen con una frecuencia más alta en el grupo de diahéticos más antiguos. Figura N. 2.

En la Tabla N." 4 aparecen los resultados de los EEG y las glicemias realizadas previamente. Se aprecia que las diferentes curvas electroencefalocráficas de distribuyen en igual proporción en los tres rangos de glicemia establecidos.

Del análisis de los antecedentes de quetoacidosis diabética y los distintos tipos de EEG, se desprendiś que el haber presentado esta complicacion melabólica en la evolución de la diabetes no influye en la distribuciun de los diversos Irazados electroencefalográficos.

Algo similar se encontró al relacionar los resul- $\bar{x}$ tadus de los EEC; y el grado de control metabuilico habitual; en efecto, los tipos de EEG se distribuyen uniformemente entre aquellos con buen control. regular " fransamente malo.
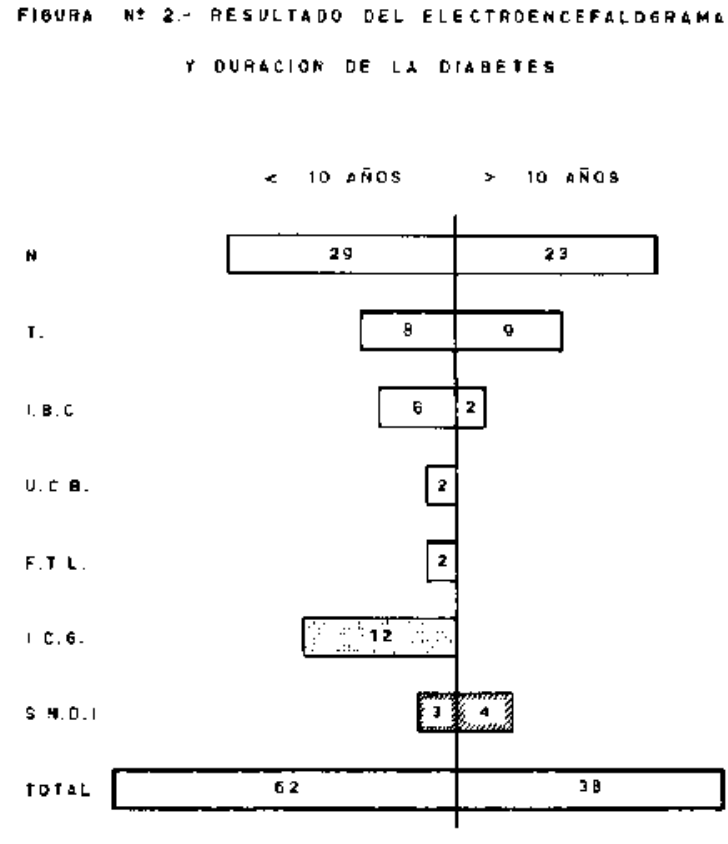

N: JE CASOS

Tabla N. ${ }^{\circ} 3$

Resultado del electroencefalograma y edad actual.

\begin{tabular}{|c|c|c|c|c|c|c|c|}
\hline \multirow{3}{*}{ Electroencefalograma } & \multicolumn{7}{|c|}{ Años de edad } \\
\hline & \multicolumn{2}{|c|}{10} & \multicolumn{2}{|c|}{$11-20$} & \multicolumn{2}{|c|}{$21-35$} & \multirow{2}{*}{$\begin{array}{c}\text { Total } \\
\text { N:o }\end{array}$} \\
\hline & N. ${ }^{\circ}$ & $\%$ & N." & $\%$ & N." & $\%$ & \\
\hline Nonnal & 4 & 31 & 3.3 & $5 \vec{i}$ & 15 & 52 & 52 \\
\hline Tensional & 2 & 15 & 6 & 10 & 9 & 31 & 17 \\
\hline Inmadurez bioeléctrica cerebral & 2 & 15 & 6 & 10 & 0 & 0 & 8 \\
\hline Umbral convulsivante bajo & 0 & 0 & 2 & 4 & 0 & 0 & 2 \\
\hline Foce temporal lento & 0 & 0 & 2 & 4 & 0 & 0 & 2 \\
\hline $\begin{array}{l}\text { Intercrítico de comicialidad } \\
\text { generalizada }\end{array}$ & 5 & 39 & 6 & 10 & 1 & 3 & 12 \\
\hline $\begin{array}{l}\text { Sufrimiento neuronal difuso } \\
\text { imespecífico }\end{array}$ & 0 & 0 & 3 & 5 & 4 & 14 & 7 \\
\hline Total & 13 & 100 & 58 & 100 & 29 & 100 & 100 \\
\hline
\end{tabular}

Tabla N. ${ }^{\circ} 4$

Resultado del electroencefalograma y glicemia previa.

\begin{tabular}{|c|c|c|c|c|c|c|c|}
\hline \multirow{3}{*}{ Electroencetialogramit } & \multirow{2}{*}{\multicolumn{2}{|c|}{$100-150$}} & \multicolumn{4}{|c|}{ Glicemia mg \% } & \multirow{3}{*}{$\begin{array}{c}\text { Total } \\
\mathrm{N}^{\prime \prime}\end{array}$} \\
\hline & & & \multirow{2}{*}{$\begin{array}{l}151 \\
\text { N." }\end{array}$} & \multirow{2}{*}{$\begin{array}{r}-250 \\
\%\end{array}$} & \multicolumn{2}{|c|}{$251 y+$} & \\
\hline & N." & $\%$ & & & N. ${ }^{\circ}$ & $\%$ & \\
\hline Normal & 10 & 53 & 26 & 57 & 16 & 46 & 52 \\
\hline Tensinnal & 4 & $2 \mathrm{I}$ & 7 & 15 & 6 & 17 & 17 \\
\hline Imuadırez biselèctrica cerebral & 2 & 10 & 2 & 4 & 4 & 11 & 8 \\
\hline Limbral convulsivante bajo & 0 & 0 & 1 & $\mathbf{z}$ & 1 & 3 & 2 \\
\hline Fiken temporal lento & 0 & 0 & 1 & 2 & 1 & 3 & 2 \\
\hline Intercritico de comicialídad & & & & & & & \\
\hline generallizikdat & 3 & 16 & 5 & 11 & 4 & 11 & 12 \\
\hline $\begin{array}{l}\text { Sutrimiento neuronal difioso } \\
\text { interpecilies) }\end{array}$ & t) & (1) & 4 & 9) & 3 & 9 & $\vec{t}$ \\
\hline Iiutal & 19 & 100 & 40 & 100 & 35 & 100 & 100 \\
\hline
\end{tabular}


Siguiendo con el análisis de nuestros hallazgos. presentamos en la Tabla N." 5 la distribución de los electroencefalogramas en relación al antecedente de hjoglicemia. El $9 \%$ no presentó hipoglicemia, el $32 \%$ tuvo hipoglicemia leve o moderada ocasional y el $59 \%$ hipoglicemia leve o moderada frecuente. En los tres subgrupos, alrededor de un $70 \%$ de los EEG fueron normales, considerando como tales a los Normales y Tensionales.

Los EEG que mostraban S.VDI se presentaron exclusivamente en los pacientes con hipoglicemia leve o moderada frecuente, pero sin significancia estadística y constituyen el $12 \%$ de los EEG de este subgrupo. La Comicialidad se reparte en forma más o menos semejante en los tres subgrupos analizados.

Én la Tabla N." 6 se estudió la influencia del antecedente de convulsiones hipoglicémicas, ubservándose que éstas no guardan relación con los tipros de EEC.

Se analizaron los hallazgos electroencefalográficos según la existencia previa de shock hipoglicémico (Tabla N."7), observándose que los EEG com Sulrimiento Veuronal Difuso Inespecifico se presentan en un porcentaje mayor en los que habian tenido hipoglicemia con pérdida de ronciencia (12\%) comparado con aquellus que no presentaron shock ( $4 \%$ ); sin embargo, esta diferencia no es estadisticamente significativa.

\section{Tabla N.0 5}

Resultado del electroencefalograma y antecedentes de hipoglicemia - síntomas leves y moderados.

\begin{tabular}{|c|c|c|c|c|c|c|c|}
\hline \multirow{2}{*}{ Electroencefalograma } & \multicolumn{4}{|c|}{ Hipoglicemia } & \multicolumn{2}{|c|}{ Frecuente } & \multirow{2}{*}{$\begin{array}{c}\text { Total } \\
\text { N." }\end{array}$} \\
\hline & N." & $\%$ & N. & $\%$ & N." & $\%$ & \\
\hline Normal & 6 & 67 & 16 & 50 & 30 & 51 & 52 \\
\hline Tensional & 0 & 0 & 6 & 19 & 11 & 19 & 17 \\
\hline Inmadurez biceléctrica cerebral & 1 & 11 & 4 & 12 & 3 & 5 & 8 \\
\hline Umbral convulsivante bajo & $\mathbf{l}$ & $1 \mathrm{l}$ & 0 & 0 & 1 & 1 & 2 \\
\hline Foco temporal lento & 0 & 0 & 0 & 0 & 2 & 3 & 2 \\
\hline $\begin{array}{l}\text { Intercritico de comicíalidad } \\
\text { generalizada }\end{array}$ & I & 11 & 6 & 10 & $=$ & 0 & 8 \\
\hline $\begin{array}{l}\text { gemeranizada } \\
\text { Sutrimiento neuronal difuso }\end{array}$ & 1 & 11 & 0 & 19 & 2 & 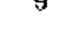 & 12 \\
\hline inespecifico & 0 & 0 & 0 & 0 & 7 & 12 & 7 \\
\hline Total & $y$ & 100 & 32 & 100 & 59 & 100 & I0k \\
\hline
\end{tabular}

\section{Tabla N. ${ }^{\circ} 6$}

Resultado del electroencefalograma y antecedentes de hipoglicemia - convulsiones.

\begin{tabular}{|c|c|c|c|c|c|}
\hline \multirow{3}{*}{ Electrotencefalograma } & & & \multicolumn{3}{|c|}{ Convulsiones } \\
\hline & \multicolumn{2}{|c|}{ Ausentes } & \multicolumn{2}{|c|}{ Presentes } & \multirow{2}{*}{$\begin{array}{c}\text { Total } \\
\text { N." }\end{array}$} \\
\hline & $\mathbf{N} .^{0}$ & $\%$ & $\mathcal{N}: "$ & $\%$ & \\
\hline Vurmal & 38 & 52 & 14 & .52 & 52 \\
\hline Tensional & 13 & Is & 4 & 15 & 17 \\
\hline Inmadurez bioeléctrica cereloral & 6 & 8 & 2 & 7 & 8 \\
\hline Untural etomonlsivante & 2 & 3 & 0 & 0 & 2 \\
\hline$F(x \cdot b$ tenujumal lentus & 1 & 1 & 1 & 4 & 2 \\
\hline $\begin{array}{l}\text { Intereritien ede corricialjolinal } \\
\text { generalieada }\end{array}$ & 8 & [ ] & 4 & 15 & 12 \\
\hline 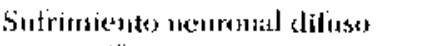 & & & & & \\
\hline incspecifics & 5 & $\vec{r}$ & 2 & $\vec{i}$ & 7 \\
\hline liot.d & 73 & {$[(x)$} & $2 \vec{i}$ & ]1MI & $\ln (N)$ \\
\hline
\end{tabular}


Resultado del electroencefalograma y intecederntes de hipoglicemia -shock.

\begin{tabular}{|c|c|c|c|c|c|}
\hline \multirow{3}{*}{ Electroencetialogrimat } & & & \multicolumn{3}{|c|}{ Sloock hipogglicentuicos } \\
\hline & \multicolumn{2}{|c|}{ Alisente } & \multicolumn{2}{|c|}{ Presente } & \multirow{2}{*}{$\begin{array}{l}\text { Tutal } \\
\text { N.". }\end{array}$} \\
\hline & $\therefore 0$ & $\%$ & N." & $\%$ & \\
\hline Normial & 31 & 54 & 21 & 50 & 52 \\
\hline Tensiontal & (6) & 17 & 7 & $1 \bar{i}$ & 17 \\
\hline Inmadure bioclectrica cerebral & 6 & 10 & 2 & 5 & 8 \\
\hline U'mbral convulsivamte bajo & 2 & 4 & 0 & 0 & 2 \\
\hline Foco temporal lento & I & 2 & 1 & 2 & 2 \\
\hline $\begin{array}{l}\text { Intereritico de comicialidad } \\
\text { generalizada }\end{array}$ & fi & 10 & 6 & 14 & 12 \\
\hline Sufrimiento neuronal difinso & & & & & \\
\hline inespecifico & 2 & 4 & 5 & 12 & 7 \\
\hline
\end{tabular}

\section{DISCUSION}

Del análisis de nuestros resultados encintramos que en los diabéticus, el $81 \%$ de los FEG eran nurmales 0 dentro de limites normales, cifra superior a otros autires. (3-B) Sin embargo, en el grupm-tontrol el $100 \%$ se encuntrí dentro de limites normales.

La Inmadurez Bineléctrica Cerelıral se presentí tanto en los diabéticos como en los contrales, sólo en los menores de 20 añus, situacion fisiologica a estas edades, en que aún puede no estar completa la madurez biocléctrica cerebral.

EEG francamente alterados se observaron en el 19\% de los casos entudiados (12\% Intereriticos de Comicialidad Generalizada y el $7 \%$ Sufrimientı Veuronal Difusoi. Este $12 \%$ de Cumicialidad es absolutamente comparable al $11 \%$ encontrado por Eeg-Olıfsson, "6" quien los clasificó como de actividad paroxistica. El mismo autor, en un trabajo anterior, ${ }^{8}$ ' estudiando diabéticus con un promedio de edad inferior al nuestro, encontró un $8 \%$ de EEG con este mismo tipo de daño cerebral.

Debemos destacar que los trazados con características de Comicialidad se presentan en los juveniles de menor edad promedio $(12,5$ años $\pm 4,5\}$ y con pocos años de enfermedad (4 años $\pm 2,4$ ). no hubo ningún caso en nin̄os con más de 10 años de diabetes, lo que hablaría en el sentido que esta alteración electroencefalográfica es independiente de la antigüedar de la diabetes y del trastorno metabólice. Esto mismo ha sido confirmado ya por otros autores. ${ }^{3-6}$ quienes incluso sugieren la existencia de una encefalopatia diabetica primaria, depen- diente tal vez de factores genéticos. Más aún. Heik. citado por Jakobson, 151 ha demostrado curvas patológicas en algunos diabéticos juveniles de diagnóslico muy recionte. en los cuales no hay tiempo sufiriente para atribuir estas anormalidades a un efecla deletéreo de la diabetes.

Nos resulta dificil entender que no tengamos en nuestra casuistica EEG del tipo ICG en pacientes an más de 10 años de enfermedad, puesto que al presentarse precozmente en la crolusión de la diabetes. deberían persistir. Tal vez podría tratarse de una anormalidad transitoria o reversible, que posteriormente tendiera a normalizarse. Fsta interpretación está en purte avalada por et trabajo de Halonen.! 4 , quien en un estudio de seguimiento electroencefalográficu) durante 5 años, en 198 diabéticos juveniles. observis grandes cambios a través del tiempo y en 36 casos tenían EEG alterado inicial; éstos se normalizaron posteriormente.

Debernos destacar que el Sufrimiento Neuronal Difuso Inespecítico, que aleanzó al $7 \%$, se presentó sólo en los mayores de 10 años de edad y, aun cuando las diterencias no son estadísticamente significativas por el número bajo de pacientes, muestran una tendencia muy clara en el sentido de que esta anormalidad aparece sólo en diabéticos juveniles de más edad. Esta aseveración es confirmada por el alto promedio de edad de este grupo $(25,9$ años $\pm 7,4)$. El mismo hecho se observa al relacionar esta alteración electroencefalográfica y la duración de la diabetes, ya que estos pacientes tenían una larga evolución de su cuadro metabólico (13,3 años $\pm 7,9)$.

Al igual que otros autores, ${ }^{5-31}$ no encontra- 


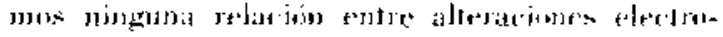

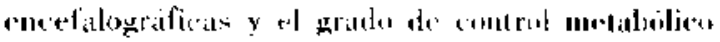

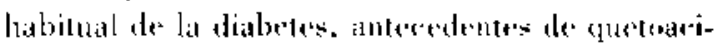

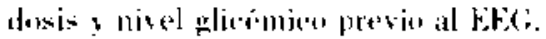

Cambera logice de toperar en diabetions juver-

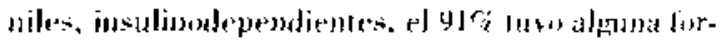

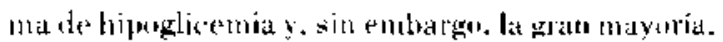
$81 \%$ de los pacientes. avierun FEt, dentro de limites mormakes.

Los trazadir que demestramon Comiclialidad no

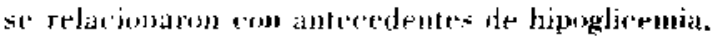

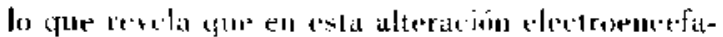

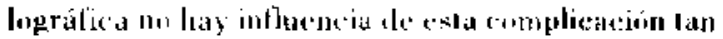
habitual del tratamiento insulinico. Fat berbo ya ha

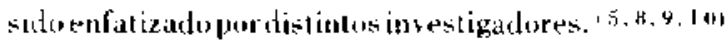

Llamri la atemedrin gue no observamoss SNDI en

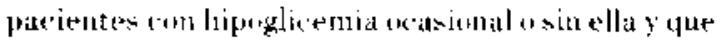
las 12 castos se presentaron ancociadas a hipoglicesmias leves om moderadas peru frecuentes. Fstu hace plantear la hiputesis que esta manifestarion electroencefalengufica estuviera iondicionada, ers parte. por hipoglicemias repetidas, pere mo influenciada por consulsiones hipoglicemicas "shoke. ya que no hubs relacioin entre estas dos cilimas formas y el suifimiento neuronal qus eomentamos.

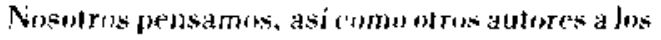
pue ya hemos herhe mención. pue la Comicialidad es independiente de la diabetes y del tratamiento insulínico, probablemente gentis'a. pero ereems que en el daño difuss neuronal puede hatoer (jerta participasión del fenomejo hipoglicemicu. Para aclarar esta hipotesis es necesario realizar un estudio semejante en diabélicus jureniles, antes que inicien el tratamiento insulítico, investigación que no hemos encontrado en la literatura a nuestro aloasce.

\section{RESL MEN Y CONCIUISIONES}

Se realizi EEG en 100 diabétions juveriles (39) humbres y 61 mujerest elegidos al azar entre los atendidos habitualmente en nuestru Departamento y en 50 controles. El principal ubjetivo fue deterninar algura posible relacioin entre antecedentes de hipoglicemia y alteraciones electruencefalograficas.

Se practicó encuesta sobre hipuglicemias anamnisticas, encontrándose que el $91 \%$ de los c*asus habian presentado algún grado de hipoglicemia.

A continuación se realizó el EEG en todos los pacientes con niveles glicémicos superiores a $100 \mathrm{mg} \%$ y lus trazados electroencelalográficos revelaron los siguientes resultados: Normales $52 \%$.

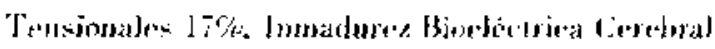

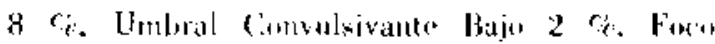

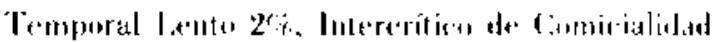

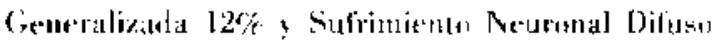

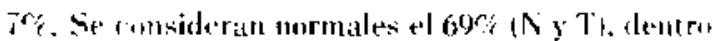

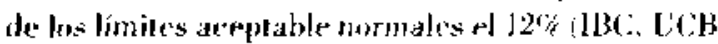

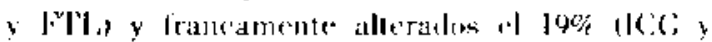

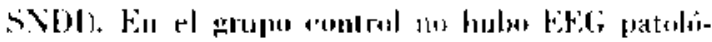
aicos.

E.l 70\% de los pacientes que prenentaran hipngli-

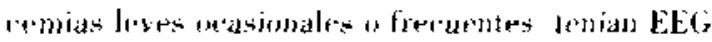
nommal. La Inmadurez Bineleceriea Cerebral, en

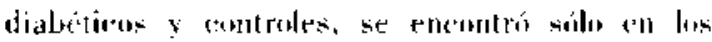
menures de 20 años de edad.

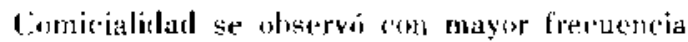
(39\%) en pactionles menores de 10 añes de tedad y con paros arion de evolucion de la diabetes $(4$ años $\pm 2,4)$

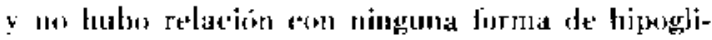
('enia anamméstica.

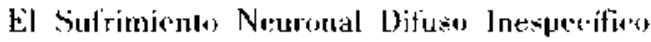
aparecio en situte pacientes. todos ellos mayores de 11 añus de rdad y an un proréentaje mats allo en lis maycres de 21 añis (l4?\%). La antigüedad promedio, do la diabeles de estors enfermes alcanan a 13.3 años $=7.9$. Thodos tenian antecedentes de hipoglicemias leves frecuentes y cines de ellos habian presentado shork hipogliermico. La existencia de (convulsimes hipoglic émicas no se ascoció con este tipo de E.FC.

Nib hubo relacioin entre las glitemias previas al EFC y los resultados do śste, eromo lampureo con el grado de romlrol metabilieo habitual del paevente.

De acuerdn a este esludion. podemos conchuir que la mayoría de los diabéticos juveniles investigadis presentan un EEG dentro de los límiles nornales. Ia duraciún de la diabetes no intluye en las alteraciones del EEC de tipo Intercritico do Comichalidad Generalizada, como tamposo lo hacen las diversas formas de hipoglicemia.

El Sufrimiento Nauronal Difuso Inespecifico se presenta a mayor edad y antigüedad de la diabetes y parcer ser. a lo mems en parte, favorerido por distintas [ormas de hipoglicemia.

\section{REFERENCIAS}

I Christian, WW. "Atlas de Electruent*fahogralia C'linita". Ed. F'az Mantalvo. Madrid, 1971.

2 Harble. A., White, F., Brodleb, R. and Krall, 1. "Juslin's Diabetes Mellitus. Lea and Vebiger. Philadelphia, 1971.

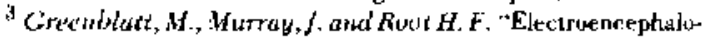
graphic Studies in Diabetry Mellitus". New Enzland J. Med. 234: 119.1446. 
4 Gardner, L, and Reyerbach, G. C. "Brain Domage in a Juvenile Diabelic Patient Agsociated wilh Insulin Hypoglycemia". Pedietrics 7: 210. 1951.

5 Jakabron, T., Huhmur, E. and Rimon, R. "Electroencephalographạc Alierations in Diabetes Mellitue". Acta Med. Scand. $180: 41,1966$.

${ }^{6}$ Eeg-Olofsson, 0. "Hypoglycemia and Neurological Disturbatiuns in Children with Diabetes Mellitus". Acta Paediatric seand. Suppl. 270: 91, 197 ?.

${ }^{7}$ Mella, L., Garcia de los Rtos, M., Lopez, G. y Schilling, E.
"Diabetes Infantil". Rev. Chil. Ped. 44: 207, 1973.

${ }^{B}$ Eeg-Olofson, O. and Petersen, I. "Childhood Diabetic Neuroparhy. A. Clinical and Neurophysiological Study". Acta Paediatr. Scand. 55: 163, 1966.

${ }^{9}$ Halonen, H., Hiekkala, H., Huupponen, H. and Häkkinen, V. A. Follaw-up EEG Siudy in Diabetic Children". Electroenceph. Clin. Neurophysiol. 43: 519, 1977.

10 Fallströn, K. "On the Pergonality Structure in Diabetic Sehool Children Aged 7-15 Years". Acta Paediatr. Seand. Suppt. 251: 13. 1974. 